\title{
Complex satellite systems: a general model of formation from rings
}

\author{
Aurélien Crida ${ }^{1}$ and Sébastien Charnoz ${ }^{2,3}$ \\ ${ }^{1}$ Laboratoire Lagrange (UMR 7293) \\ Université Nice Sophia-antipolis / CNRS / Observatoire de la Côte d'Azur \\ CS 34229, 06304 NICE cedex 4, FRANCE \\ email: crida@oca.eu \\ ${ }^{2}$ Laboratoire AIM (UMR 7158) ; Université Paris Diderot / CEA / IRFU-SAp \\ 91191 Gif-sur-Yvette cedex, FRANCE \\ ${ }^{3}$ Institut Universitaire de France; 103 Bd Saint Michel, 75005 Paris, FRANCE.
}

Summary of the Abstract. We present our model of formation of regular satellites by the spreading of a cold debris disk beyond the Roche radius. By numerical simulations and analytical calculations, we show that this process explains the peculiar properties of Saturn's small and mid-sized moons, in particular their mass - distance distribution. This process seems to also account for the structures of the satellite systems of Uranus, Neptune, and the Earth, suggesting that they used to have massive rings.

\begin{abstract}
Satellite systems are often seen as mini-planetary systems, and they are as various and complex. The Earth has one single satellite of more than one percent of its own mass, but the giant planets have many, some of them tiny. They constitute fascinating, interacting, evolving, and dynamically complex systems. While irregular satellites, having inclined and eccentric orbits, are supposed to be captured, it was generally admitted that regular satellites, having circular orbits in the equatorial plane of their host planet, formed in the gas and dust circum-planetary disk that surrounded giant planets at the time of their formation, more or less similarly as planets formed in the proto-planetary disk around their host star. Here, we propose an alternative mechanism for the formation of regular satellites: the spreading of a dynamically cold disk of solids beyond the Roche radius of a planet.

The Roche radius is defined as the distance from a planet beyond which self-gravity is stronger than tidal forces, allowing gravitationally bound aggregates to form, and satellites to exist. Saturn's rings are inside the Roche radius, which is why they don't coalesce into one single object. However, like any astrophysical disk in Keplerian rotation, they spread. Once material reaches the Roche limit, it should accrete into moonlets. These moonlets migrate outwards, repelled by tidal interactions with the rings and Saturn. We showed with numerical simulations that the small and mid-sized moons of Saturn formed this way, which explains their young age and composition (Charnoz et al. 2010, 2011). Analytical calculations show that the formation of satellites from a ring of solids spreading beyond the Roche limit takes place in 2 phases: (i) the continuous regime, in which only one satellite forms (this regime prevailed for the moon-forming disk around the Earth) ; (ii) the pyramidal regime, in which a series of moons form, migrate outwards, merge... In this regime, the satellites should follow a precise mass-distance relation, which matches that of the Saturnian system, confirming that it formed this way. It also fits the Uranian and Neptunian systems, suggesting that these planets used to have massive rings that gave birth to their regular satellites, and vanished (Crida \& Charnoz (2012)).

Refinements of this model, applications to planetary systems, and a recent likely observation of this process will be discussed in conclusion.
\end{abstract}

Keywords. planets and satellites: formation, planets and satellites: individual (Saturn), planets: rings 

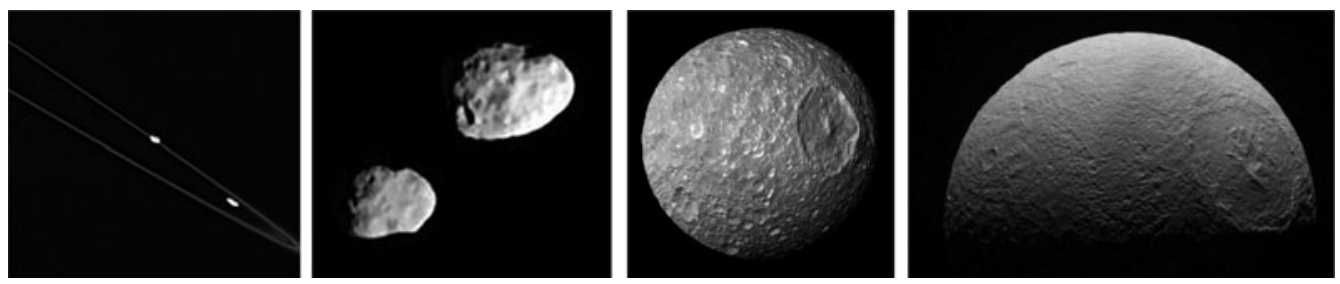

Figure 1. Pictures of Saturn regular satellites, by the Cassini spacecraft. From left to right: Prometheus and Pandora around the F-ring; Janus and Epimetheus; Mimas; Tethys.

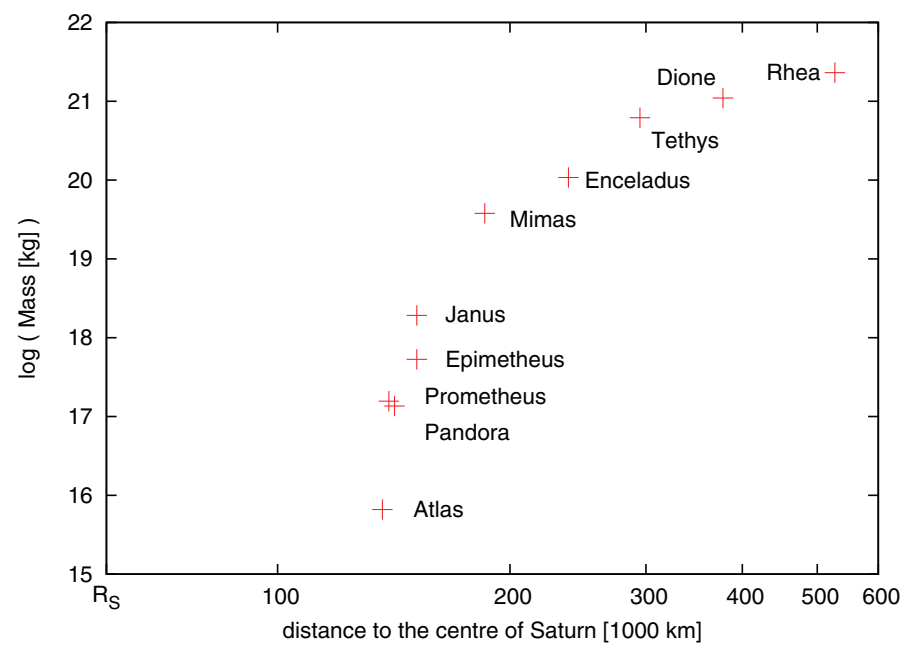

Figure 2. Saturn's regular satellites in a mass - distance diagram (logarithmic scale). A smooth increase of the mass with distance, starting from 0 at $140000 \mathrm{~km}$ from Saturn's centre, appears clearly. The + are just symbols, not errorbars.

\section{Introduction}

The regular satellites of Saturn are much better known since the Cassini spacecraft brought amazing pictures of these icy worlds (Figure 1). Starting at the outer edge of the rings (140 $000 \mathrm{~km}$ from Saturn's centre), one finds Prometheus and Pandora on both sides of the F-ring, who are tiny icy bodies, of about $80 \mathrm{~km}$ in size and $10^{17} \mathrm{~kg}$. Eleven thousand kilometres further, Janus and Epimetheus share the same orbit, for a total mass of $2.5 \times 10^{18} \mathrm{~kg}$. Then, Mimas stands at $185000 \mathrm{~km}$ from Saturn's centre, and is roughly $400 \mathrm{~km}$ in size and $3.7 \times 10^{19} \mathrm{~kg}$. Moving away from Saturn, Enceladus, Tethys, Dione, and Rhea are ranked in size and mass as well, the latter being $390000 \mathrm{~km}$ from the edge of the rings with a mass of $2.3 \times 10^{21} \mathrm{~kg}$. Finally, the main satellite, Titan, has an orbital radius of $1.2 \times 10^{6} \mathrm{~km}$ and a mass of $1.3 \times 10^{23} \mathrm{~kg}$. In fact, as shown on Figure 2, there is an intriguing mass - distance relation: a smooth increase of the mass with distance, starting from 0 at $140000 \mathrm{~km}$ from Saturn's centre, id est from the Roche radius at the outer edge of the rings. An other noticeable property of this system is that the further from Saturn, the further satellites are from each other.

A similar trend, although less clean, is observed in the regular satellite systems of the other giant planets. Such a remarkable property can't be explained in the frame of satellite formation inside a circum-planetary disc of gas and dust around the young giant planet. In contrast, the accumulation of small bodies just outside the rings suggest that rings and satellites are somehow connected. 


\section{Evolution of rings}

Saturn's rings are made of ice blocks on circular orbit in the equatorial plane of the planet. They constitute a dynamically cold debris disk. Their velocity dispersion is so small that the thickness of the rings is about ten millions times smaller than their radius. In fact, encounters between ice blocks happen all the time, at tiny velocity. The only reason why they don't coalesce into a gravitationally bound aggregate is that Saturn's tidal forces are stronger than the self-gravity of the blocks. But tidal forces weaken with the distance to the planet. The distance at which tides become weaker than self-gravity is called the Roche radius, and given by:

$$
r_{\text {Roche }}=\left(\frac{9 M_{p}}{\pi \rho}\right)^{1 / 3},
$$

where $M_{p}$ is the mass of the planet, and $\rho$ the volume density of the blocks (see for instance Crida \& Charnoz (2013) ). Around Saturn, for the density of porous ice $\left(600 \mathrm{~kg} / \mathrm{m}^{3}\right)$, $r_{\text {Roche }}=140000 \mathrm{~km}$. Inside the Roche radius, aggregation is impossible, and the rings have to stay as rings.

In Keplerian rotation around a central body, the angular velocity $\Omega$ decreases with the orbital radius $r$, as given by Kepler's law : $\Omega=\sqrt{G M_{p} / r^{3}}$, where $G$ is the gravitational constant and $M_{p}$ the mass of the central body. Nonetheless, the specific angular momentum $j=r^{2} \Omega \propto \sqrt{r}$ increases with $r$. Interactions between the fast inner particles and the slow outer particles slow down the first ones, and accelerate the second ones (this can be modeled as a viscous friction). As a consequence, the inner particles lose angular momentum and move to smaller $r$, while the outer ones gain angular momentum and move to larger $r$. The rings spread, while angular momentum flows outwards. This is a well-known result, and has been described for instance by Lynden-Bell \& Pringle (1974).

Using a prescription for the viscosity of Saturn's rings given by Daisaka et al. (2001), Salmon et al. (2010) have computed the evolution of the density profile of the rings with a 1D implicit code. They find that the more massive the rings are, the faster they spread. Two major results derive from their analysis: (i) with their present mass, Saturn's rings can survive for billions of years, thus they can be as old as the Solar System; (ii) they could have been much more massive in the past and lost mass by spreading.

According to Daisaka et al. (2001), the viscosity $\nu$ of massive rings is proportional to their mass squared. More precisely, the characteristic time for viscous spreading is $t_{\nu}=r_{\text {Roche }}^{2} / \nu=\frac{\pi}{92} D^{-2} T_{R}$, where $D=M_{\text {rings }} / M_{p}$ and $T_{R}$ is the orbital period at $r_{\text {Roche. As }} d D / d t=-D / t_{\nu}$, one gets $d D / d \bar{t} \approx-30 D^{3}$, with $\bar{t}=t / T_{R}$. The solution of this differential equation is :

$$
D(t)=\frac{1}{\sqrt{60 \bar{t}+D_{0}^{-2}}} .
$$

Clearly, the initial condition $D_{0}$ is erased as soon as $\bar{t} \gg 1 / 60 D_{0}^{2}$, and on long term, $D(t) \approx(60 \bar{t})^{-1 / 2}$. Around Saturn, with $t=4.5$ billon years, this gives $D=8 \times 10^{-8}$.

Actually, Canup (2010) suggests that the rings formed from the tidal stripping of a differentiated satellite that migrated inside the Roche radius of Saturn (see also Crida \& Charnoz (2010)). In that case, the rings were a few times $10^{22} \mathrm{~kg} 4.5$ billion years ago, while they are only $\sim 4 \times 10^{19} \mathrm{~kg}$ now $\left(D=7 \times 10^{-8}\right.$, in agreement with the estimate above). 


\section{The satellites of Saturn are children of the rings}

The rings spread. Material that falls onto Saturn is lost forever, but what about material spreading outwards, beyond the Roche radius ? As soon as this limit is crossed, self-gravity wins over tidal forces. Aggregates form, and new small satellites are produced at $r_{\text {Roche }}$. These satellites have a smaller angular velocity than rings particles; hence, they are accelerated by them, gain angular momentum, and move further. Lin \& Papaloizou (1979) have computed the torque felt by a satellite of mass $m$ orbiting on a circular orbit of radius $r$ with angular velocity $\Omega$ outside of rings of surface density $\Sigma$ :

$$
\Gamma=\frac{8}{27}\left(\frac{m}{M_{p}}\right)^{2} \Sigma r^{4} \Omega^{2} \Delta^{-3}
$$

where $\Delta=\left(r-r_{\text {Roche }}\right) / r_{\text {Roche }}$ is the normalised distance to the outer edge of the rings.

Applying this to Janus, one finds that it is has been moving away from $r_{\text {Roche }}$ for $\sim 10^{8}$ years. In other words, a hundred million years ago, Janus must have been inside the rings. This agrees with the observation that Janus is little cratered, and its surface looks much younger than the Solar System. In fact, Janus, Epimetheus, Prometheus and Pandora share this young aspect, and are all under-dense $\left(\sim 600 \mathrm{~kg} / \mathrm{m}^{3}\right)$, and have the same spectrum as the rings. All this suggests very strongly that they have been formed recently, from the spreading of the rings.

Charnoz, Salmon \& Crida (2010) performed numerical simulations of the evolution of present day rings, similar to Salmon et al. (2010), but with the mass falling beyond $r_{\text {Roche }}$ being transformed in small satellites whose evolution is governed by Eq. (3.1). The small satellites are allowed to merge, and the final outcomes of their simulations is in general a system of a handful of satellites of masses in agreement with that of the small moons.

From this spectacular result, it is tempting to generalise to the whole system of regular moons of Saturn. There is a problem though : beyond the 2:1 Mean Motion Resonance with $r_{\text {Roche }}$, located at $222000 \mathrm{~km}$ from the centre of Saturn, the interaction with the rings vanishes. The only process that makes the satellites migrate is the dissipation of tides inside Saturn, with the rate:

$$
\frac{d r}{d t}=\frac{3 k_{2 p} m \sqrt{G} R_{p}^{5}}{Q_{p} \sqrt{M_{p}} r^{11 / 2}}
$$

where the index $p$ refers to the planet Saturn, $k_{2}$ and $Q$ are the Love number and the dissipation factor respectively. With the old value of $Q_{\text {Saturn }} \approx 18000$, this gives a too small rate, and Enceladus, Tethys, Dione and Rhea don't migrate significantly over the age of the Solar System; so they must have been formed at their present location. However, a careful analysis of old photographic plates performed by Lainey et al. (2012) reveal that their migration is detectable over a century, and that $Q_{p}$ must be 10 times smaller. With $Q_{p}=1700$, the time it takes for Rhea to migrate from $222000 \mathrm{~km}$ to its present position is about 3.5 billion years. Such a "tidal age" can be calculated for the other mid-sized moons, and they appear to be ranked by increasing tidal age (Charnoz et al. (2011)). This opens the possibility of forming Rhea first, then Dione, then Tethys, then Enceladus, and finally Mimas, and have them migrate to their present orbit within the age of the Solar System, without orbit crossing.

Starting simulations with very massive rings as suggested by Canup (2010), using Eq. (3.1) inside $222000 \mathrm{~km}$ and Eq. (3.2) with $Q_{p}=1700$ beyond this, Charnoz et al. (2011) reproduce successfully the system of the regular satellites of Saturn up to and including Rhea. 
Not only is this model in agreement with the mass - distance distribution of the system of the regular satellites of Saturn, but this also solves two problems at the same time. (i) These moons have young crater ages, and anyway should not have survived the Late Heavy Bombardment; forming them not earlier than 3.9 billions years ago is therefore very positive. (ii) These moons have rocky cores, which account for $7 \%$ to $70 \%$ of the mass of the body; these variations in the bulk composition is hard to explain in a gaseous circum-planetary disk, and the differentiation itself is unexpected for such small objects. In this model, chunks of silicates initially present in the rings coalesce into a handful of rocky cores (because the Roche radius for silicates is only $90000 \mathrm{~km}$ ), and are later coated with ice from rings; this naturally forms differentiated bodies, of stochastic bulk composition (Charnoz et al. (2011)).

\section{A general, analytic model}

Crida \& Charnoz (2012) describe analytically the spreading of rings beyond the Roche radius, as studied before with numerical simulations. We define

$$
\tau=M / F T_{R}
$$

is the dimensionless lifetime of the rings, where $M$ is their mass, $F$ is the flow of mass through the Roche limit, and we remind that $T_{R}$ is the orbital period at $r_{\text {Roche }}$ and $D=M / M_{p}$. With Daisaka et al. (2001)'s prescription for the viscosity, $\tau=r_{\text {Roche }}^{2} / \nu T_{R}=0.0425 / D^{2}$.

\subsection{Continuous regime}

Assume one satellite forms first at $r_{\text {Roche }}$; its mass is then $m=F t$ with $t$ the time since its birth. Note $q(t)=m(t) / M_{p}$ the satellite to planet mass ratio. As it grows, it migrates, following Eq. (3.1). One can then show easily that:

$$
q=\left(\frac{\sqrt{3}}{2}\right)^{3} \tau^{-1 / 2} \Delta^{2}
$$

We call this the continuous regime, in which the satellite's mass increases linearly with time, while its distance to the rings $\Delta$ increases as the square root of the time.

This regime is applicable if and only if the Roche limit, where ring material is delivered, falls within the feeding zone of the satellite. This feeding zone is 2 times its Hill radius $r_{H}=(q / 3)^{1 / 3} r$; the condition for the continuous regime to apply reads therefore $\Delta<$ $2(q / 3)^{1 / 3}$. Input into Eq. (4.1), this gives :

$$
\begin{aligned}
\Delta<\Delta_{c} & =\sqrt{\frac{3}{\tau}} \\
q<q_{c} & =\frac{3^{5 / 2}}{2^{3}} \tau^{-3 / 2} .
\end{aligned}
$$

Starting at $\Delta=q=t=0$, we are in the continuous regime. Hence, as rings begin to spread beyond the Roche radius, one single satellite forms and gathers all the mass.

The smaller $\tau$ is, the larger this satellite can become; in the limit of very fast spreading, $q_{c} \geqslant D$ and only one satellite forms, whose mass is that of the rings. In the case of Saturn's rings though, $q_{c} \ll D$ and the rings are almost unaffected at the end of the continuous regime. 


\subsection{Discrete regime}

When the satellite reaches $\Delta_{c}$ ( or $q_{c}$ ), it doesn't accrete material from the rings anymore, but keeps migrating outwards at constant mass. The material flowing through $r_{\text {Roche }}$ forms a new satellite at $r_{\text {Roche }}$. This new satellite migrates outwards and is accreted by the first one. Another new satellite forms, that is soon accreted by the first one, and so on. In this regime, the first satellite still grows at the same average rate, but step by step, by accreting moonlets formed at the outer edge of the rings. We call this the discrete regime. Similarly as before, this holds as long as $\Delta<\Delta_{c}+2(q / 3)^{1 / 3}$, which gives :

$$
\begin{aligned}
\Delta & <\Delta_{d}=3.14 \Delta_{c} \\
q & <q_{d}=9.9 q_{c} .
\end{aligned}
$$

Applying this to the Earth's Moon forming disc $(D \approx 0.02)$, one gets $q_{d}$ equal to the mass of the Moon. This explains why one and only one satellite formed around the Earth.

In the case of Saturn's rings, $q_{d}=10^{-18}$, so only tiny satellites form.

\subsection{Pyramidal regime}

Satellites of mass $q_{d}$ are produced by the discrete regime at $\Delta_{d}$ every $t_{d}=q_{d} M_{p} / F$. They then migrate outwards at constant mass. Hence, their migration speed decreases (because $\left.\Gamma \propto \Delta^{-3}\right)$. Consequently, they approach each other, until they enter each other's feeding zone, and merge. This leads to the formation of satellites of mass $2 q_{d}$ every $2 t_{d}$ at a specific location. The products of these mergers migrate outwards, and merge further. And so on, in a hierarchical scheme, that we call the pyramidal regime.

Using Eq. (3.1), one finds that a merger occurs (and the mass doubles) every time the distance is multiplied by $2^{5 / 9}$. Hence, in this regime, $q \propto \Delta^{9 / 5}$. In addition, the number density of satellites should be proportional to $1 / \Delta$, which explains the observed pile-up. Beyond the 2:1 mean motion resonance with $r_{\text {Roche }}$ (located at $\Delta=0.58$ ), Eq. (3.2) applies, and $q \propto r^{3.9}$.

This mass distance relation fits remarkably well with that of the Saturnian system, shown as red + symbols on figure 3 where the dashed lines follow the above law. From Pandora (bottom left corner) to Titan (top right corner), the masses of all the satellites are within $60 \%$ of the theoretical red dashed line. This is the smoking gun proving that these satellites formed from the spreading of Saturn's formerly massive rings. It suggests that maybe even Titan comes from the same process, but its position on the line could be a coincidence, as its tidal age is of the order of ten billion years.

More surprisingly, the regular satellites of Uranus and Neptune follow the same trend. In the case of Neptune, the match is almost perfect except for one outlier, Despina, apparently three times too massive... In the system of Uranus, there is much more scatter, but this is not surprising as this system is chaotic, with frequent collisions and orbit crossing (French \& Showalter (2012)). Nonetheless, the background slope is in perfect agreement with the pyramidal regime. As a consequence, we claim that Uranus and Neptune used to have massive rings, from which their regular satellites were born. In contrast with the case of Saturn, these rings almost completely disappeared, but the satellite system kept track of its formation from their spreading beyond their Roche radius. We checked that a ring of 1.5 times the total mass of the regular satellites of these planets would have $D<2 \times 10^{-4}$, so that $q_{d}$ would be tiny, and the pyramidal regime should prevail (Crida \& Charnoz (2012)). 


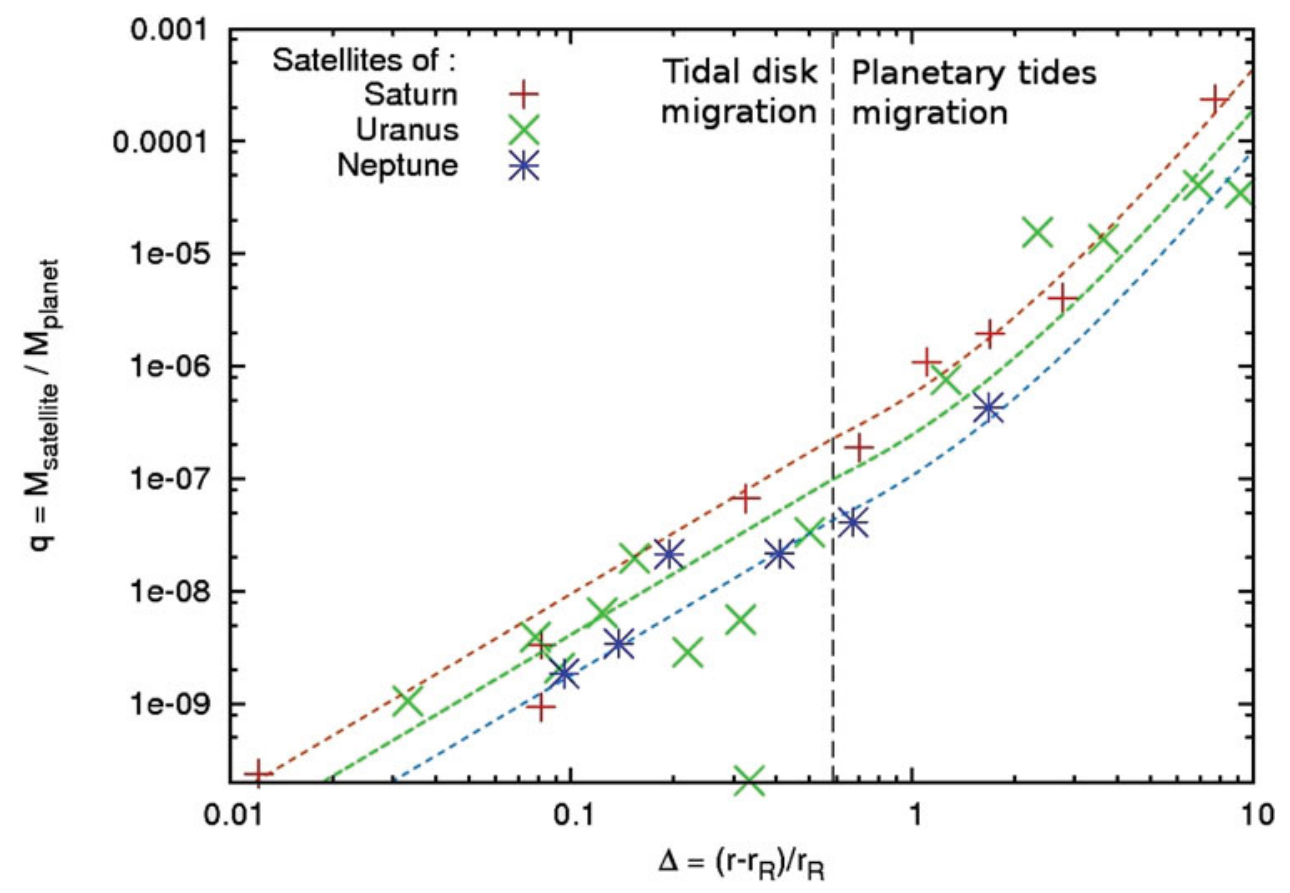

Figure 3. Symbols : Regular satellites in the $q-\Delta$ diagram for the three outer giant planets. Dashed lines : $q \propto \Delta^{9 / 5}$ for $\Delta<0.58, q \propto r^{3.9}$ for $\Delta>0.58$.

\section{Conclusion and perspectives}

We studied the spreading of a dynamically cold disk of debris (rings) beyond its Roche radius, where gravitational aggregation then takes place. At first, a single satellite forms, and migrates outwards while accreting the material that flows beyond the Roche radius (continuous then discrete regimes); then, a series of satellites are produced, whose mass increases with distance following a specific law (pyramidal regime). We have shown that this process

(a) explains the physical properties of the icy moons of Saturn,

(b) explains the mass - distance distribution of the regular satellites of the giant planets (which can be considered as an observational signature of this process),

(c) unifies terrestrial and giant planets in the same paradigm,

(d) is the likely origin of most regular satellites in the Solar System, from our Moon to the giant planets.

Although the mathematical model is complete, it relies on a few approximations, and several open questions remain.

- Jupiter's system doesn't fit in this picture. The Galilean satellites probably didn't form this way.

- Our model neglects satellite-satellite interactions, and possible resonance captures. How would this affect the pyramidal regime? Could the Laplace resonance in the Jovian system be reproduced?

- If Uranus ans Neptune had massive rings, where did they come from ? And how did they almost disappear ? These questions were, of course, never addressed...

- Could this model explain compact exoplanet systems like Kepler 32 ? One could imagine that a migrating planet gets tidally stripped by its star inside it Roche radius, and that the resulting debris disc spreads, giving birth to a second generation of planets. 
These questions open perspective for future research.

To conclude, Murray et al. (2014) have observed small bodies at the outer edge of Saturn's A ring. Their inferred size is larger than $q_{d}$ and their behaviour suggests collisions, evocative of the pyramidal regime. Future observations are needed, and the resonance with Janus will most likely perturb the behaviour of these bodies. Nonetheless, it seems that our theoretical model is possibly at play right now, and the next generation of Saturnian satellites may be built from the rings.

\section{References}

Canup, R. M. 2010, Nature, 468, 943.

Charnoz, S., Salmon, J., \& Crida, A. 2010, Nature, 465, 752.

Charnoz, S., Crida, A., Castillo-Rogez, J., Lainey, V., Dones, L., Karatekin, Ö, Tobie, G., Mathis, S., Le Poncin-Lafitte, C., \& Salmon, J. 2011, Icarus, 216, 535.

Crida, A. \& Charnoz, S. 2010, Nature, 468, 903.

Crida, A. \& Charnoz, S. 2012, Science, 338, 1196.

Crida, A. \& Charnoz, S. 2013, SF2A-2013: Proceedings of the Annual meeting of the French Society of Astronomy and Astrophysics, 57.

Daisaka, H., Tanaka, H., \& Ida, S. 2001, Icarus, 154, 296.

French, R. S. \& Showalter, M. R. 2012, Icarus, 220, 911.

Lainey, V., Karatekin, Ö., Desmars, J., Charnoz, S., Arlot, J.-E., Emelyanov, N., Le PoncinLafitte, C., Mathis, S., Remus, F., Tobie, G., \& Zahn, J.-P. 2012, ApJ, 752, 14.

Lin, D. N. C. \& Papaloizou, J. 1979, MNRAS, 186, 799.

Lynden-Bell, D. \& Pringle, J. E. 1974, MNRAS, 168, 603.

Murray, C. D., Cooper, N. J., Williams, G. A., Attree, N. O., \& Boyer, J. S. 2014, Icarus, 236, 165.

Salmon, J., Charnoz, S., Crida, A. \& Brahic, A. 2010 Icarus, 209, 771. 